Programmatische Kommentare und Repliken

Aleida Assmann*

\title{
Die Grenzenlosigkeit der Kulturwissenschaften
}


Abstract: What keeps cultural studies in motion and, more difficult still, what hold them together? They are continuously animated through so-called ,turns' that in regular intervals open up new perspectives and transform the leading issues and concepts. Such regular innovations are not only due to internal readjustments in terms of methodological changes but are also connected to cultural and social changes. In this way, cultural studies have become an integral part of the transformation of the world as we see and construct it. They are not only a lense through which we observe the transformation of the world, but also a tool with which it is produced. In this active engagement and entanglement with the real world, cultural studies have lost a sense of their professional boundaries. They are constantly extending their realm of research, incorporating avidly new territory. To the extent that cultural studies have embraced the project of cultural self-thematization and self-transformation, they have become as fluid and volatile as culture itself.

Keywords: cultural turns, translation, innovation, logic of research process, transvaluation of values, acceleration, social - epitemological - cultural change, fiction, art of the possible, concepts of the real, referent, evidence, narrative, archaeology of communism, histoire totale.

*Prof. Dr. Aleida Assmann: Fachbereich Literaturwissenschaft/Anglistik, Universität Konstanz, 78457 D-Konstanz, email: aleida.assmann@uni-konstanz.de

Während die Geisteswissenschaften im 19. Jahrhundert als historische Disziplinen entstanden, die mit dem Auftrag ausgestattet waren, sich im Nachhinein, also ausschließlich retrospektiv Gedanken über Kunst und Geschichte zu machen, ging die Wende zu den Kulturwissenschaften mit der Einsicht einher, dass ihr Diskurs zentral an der Konstitution, Konstruktion und Veränderung der Welt mit beteiligt ist. Kulturwissenschaftliche Diskurse verstehen sich in diesem Rahmen nicht nur als Reaktion auf vorgängige Ereignisse und bestehende Werke, als Beschreibung oder erklärender Kommentar im Modus der Nachträglichkeit, sondern immer öfter auch als Teil der, ways of worldmaking', als Mitwirkung an Formen der Welterschaffung und -umschaffung. Sie haben sich als eine reflexive Instanz etabliert, die kulturelle Prozesse gewissermaßen in Echtzeit beobachtet, und das Verhältnis zur Kultur, in der wir selber leben, damit um eine neue Metaebene bereichert. Doch für diese Metaebene gibt es, wie Hartmut Böhme zurecht betont hat, keinen unabhängigen Beobachterstandpunkt mehr. Damit stellt sich aber ein neues Problem: Was genau macht den Gegenstandsbereich der Kulturwissenschaften aus und wie kann man ihn ein- und abgrenzen? Meine Überlegungen zu dieser Frage gliedern sich in vier Schritte. Ich beginne mit der
Logik der ,cultural turns', um von hier aus auf allgemeinere Fragen des Kulturwandels zu sprechen zu kommen. In einem dritten Schritt gehe ich auf neue Schlüsselbegriffe in den Kulturwissenschaften ein und werde mit zwei Beispielen einer exemplarisch entgrenzten Kulturwissenschaft schließen.

\section{Zur Logik der Cultural Turns}

Die cultural turns halten die Kulturwissenschaften in ständiger Bewegung. Das hat Vor- und Nachteile. Doris Bachmann-Medick hat in ihrem Band Cultural Turns. Neuorientierung in den Kulturwissenschaften $\left(2006 /{ }^{5} 2014\right)$ sieben solcher ,turns' ausgewählt und näher beschrieben: interpretive, performative, reflexive, postcolonial, translational, spatial, iconic. Damit hat sie dankenswerterweise Übersicht in den Strudel der turns gebracht, uns aber zugleich auch ein gesteigertes Bewusstsein von der Beschleunigung wissenschaftlicher Paradigmenwechsel vermittelt. Ihr Buch, in dem sie die turns als amerikanische Importartikel vorstellte, um sie in den deutschen Kulturwissenschaften heimisch zu machen, war hoch willkommen als Überblick über neue For- 
schungs-Entwicklungen und als Orientierungshilfe für Studierende. Auf der anderen Seite kam aber auch Unsicherheit und Unbehagen auf angesichts der schnellen Abfolge dieser Richtungsänderungen. Man fragt sich: Wie wird es nach den sieben vorgestellten turns weitergehen? Es war die Rede von ,Schwindel' und gar von ,Schleudertrauma' (Bachmann-Medick 2014: 409). Darauf hat Bachmann-Medick mit ihrem Nachwort zur dritten Auflage beschwichtigend reagiert. Sie vermutet, "dass wir uns gegenwärtig wohl nicht mehr in der Hochphase eines Wende-Enthusiasmus befinden, der immer nur noch weitere Neuorientierungen in die Welt setzt. Eher befinden wir uns in der Phase der ,Übersetzung' der turns im Sinn ihrer kritischen Weiterentfaltung." (Bachmann-Medick 2014: 421)

Der Begriff ,turn' bzw. 'Wende' entstammt einer Rhetorik der Innovation, die unweigerlich den Effekt der Beschleunigung hervorbringt. Turns sind forschungsstrategisch wichtig, weil sie auf die Frage: ,Was gibt's Neues?' eine klare und pointierte Antwort bereithalten. Wer diese Frage stellt, will konkret wissen, wohin der Orientierungspfeil zeigt, wohin wir als nächstes marschieren sollen, wohin wir die Kolonnen der Drittmittelforschung leiten und was die ergiebigen Themen mit Innovationsgehalt für Qualifikationsschriften sind, die wir von den Jüngeren erwarten. Jeder turn ist eine Sache des Agenda Settings, der Deutungsmacht von Forschungsinstitutionen und der strategischen Durchsetzung von Forschungsschwerpunkten.

Was geschieht bei einer solchen Umleitung des Forschungsprozesses auf ein neues Ziel? Wenn der Schalter bei einem wissenschaftlichen Paradigma-Wechsel umgelegt wird, entsteht eine durchgreifende Umwertung der Werte, die man auch als eine Umstrukturierung von Erinnern und Vergessen beschreiben kann. Karl Schlögel hat uns daran erinnert, dass jede Innovation zu einem großen Teil durch Vergessen produziert wird. Erneuern und Veralten sind dialektisch aufeinander bezogen und nicht voneinander zu trennen. So gesehen ist das Entscheidende am Paradigma letztlich seine Auswechselbarkeit: „Wenn es soweit ist, dann ist ein Deutungsmonopol zu Ende gegangen, erodiert, abgesetzt und ein anderes an seine Stelle gerückt, ohne dass eine Spur noch auf die vorangegangenen Auseinandersetzungen, ja Kämpfe verweisen würde. Ein Kapitel ist geschlossen, ein anderes geöffnet." (Schlögel 2003: 60) Nicht unproblematisch an dieser linearen Ersetzungslogik des Paradigmenwechsels ist, dass mit jeder Wende Dichotomien produziert werden, die gemäß dem Innovationsimperativ des ,Zeitregimes der Moderne' das jeweils Alte vom jeweils Neuen abspalten. Auf diese Weise entwertet und verdunkelt zum Beispiel der spatial turn die Frage nach der Zeit, der performative turn die Ausrichtung auf Texte, der visual oder iconic turn die Bedeutung von Worten, oder der acoustic turn die Bedeutung visueller Wahrnehmung.

In den Nachworten zur dritten (2006) und fünften Auflage (2014) ihres Buches hat Bachmann-Medick zu diesen Begleitgeräuschen der Kritik und Abwehr Stellung genommen und dabei Funktion und Status der turns neu überdacht und erläutert. Sie versucht dabei, den kurzatmigen Diskurs über turns zu entschleunigen und setzt dabei statt auf schnelle Wechsel auf Verknüpfungen, Vertiefung, Erdung und Zusammenhang. Ihr Anliegen ist vor allem die Übersetzung nationaler Wissenschaftskulturen in eine transnationale Kulturforschung. Die turns eignen sich dabei als "systematische Gelenkstellen", an denen sich eine Verbindung von neuen Konzepten mit bestehenden Methoden unterschiedlicher Disziplinen vollziehen kann (Bachmann-Medick 2014: 420). Sie werden damit zu wichtigen Scharnieren und Garanten (trans-)disziplinären Arbeitens, denn sie markieren Zonen der Überschneidung unterschiedlicher Forschungszugänge. Ein Grundproblem kann Bachmann-Medick auf diese Weise allerdings nicht lösen, und das hängt mit der Tatsache zusammen, dass sie es einerseits mit Forschungsrichtungen, den turns, die eine interne Bewegung unserer akademischen Fächerdynamik abbilden oder in Gang setzen, und andererseits mit gesellschaftlichem Wandel und Problemfeldern zu tun hat, die sich mit den turns nicht so einfach gleichsetzen lassen. Es gibt nämlich zwei Arten von Wenden, die Bewegung in die Kulturwissenschaften bringen: solche, die ausschließlich innerhalb der Universitäten stattfinden, und solche, die sowohl innerhalb als auch außerhalb der Universitäten stattfinden. Lediglich den ersten Typ nenne ich ,turn'. Machen wir uns nichts vor: von ihnen nimmt die Gesellschaft im Allgemeinen in aller Regel keinerlei Notiz. Die meisten turns beziehen sich ausschließlich auf rein innerakademische Diskurse und nicht auf solche, die 
auch die breite Öffentlichkeit bewegen. Sie sind deshalb anschlussfähig an andere Diskurse (wie Bachmann-Medick betont), aber nicht unbedingt an Entwicklungen außerhalb der Universität.

\section{Cultural Turns und Kulturwandel}

Obwohl die Entstehung der Kulturwissenschaft ebenso wie die kulturwissenschaftliche Neuorientierung verschiedener Disziplinen selbst das Ergebnis eines tiefgreifenden Kulturwandels in der Moderne sind, gibt es immer wieder auch neue Fragestellungen und Problemfelder, die nicht in der Logik der turns eben jener Kulturwissenschaften aufgehen. Der Grund ist einfach: Sie sind Teil eines allgemeineren Kulturwandels. Hat sich die Kulturwissenschaft einmal etabliert, muss sie selbst wiederum Schritt halten mit dem Wandel der Kultur. Nehmen wir dafür das Beispiel ,Gender', das in der Liste der von Bachmann-Medick dargestellten turns gar nicht auftaucht. Hierzu gibt die Autorin folgende Erklärung ab: Gender könne in ihrem Buch schon deshalb nicht als kulturwissenschaftlicher turn angesehen werden, weil Gender als Analysekategorie ihre gesellschaftliche Aufladung und enge Einbindung in die amerikanischen Cultural Studies bei ihrer Übertragung in die hiesigen Kulturwissenschaften in großen Teilen verloren habe. Gleichsam ,lost in translation', wurde gender - so Bachmann-Medick (2014: 412) - "hierzulande eher abgewehrt, ausgelagert, entschärft, entpolitisiert." An dieser Formulierung wird deutlich, wie notwendig es ist, zwischen wissenschaftlichem Paradigma und kulturellem Wandel zu unterscheiden. Natürlich kann keine Rede davon sein, dass die Kategorie Gender auf ihrer Reise nach Deutschland verloren gegangen und hierzulande abgewehrt und kaum beachtet worden sei. Sie verweist vielmehr auf einen Kulturwandel, der längst tief in den gesellschaftlichen Institutionen und Kommunikationsformen verankert ist. Da hier aber noch längst nicht alles zum Besten steht, wird wohl niemand sagen können, dass diese Kategorie ihre intellektuelle Relevanz und politische Brisanz gänzlich verloren habe. Wenn überhaupt, dann trifft die Aussage also nur sehr eingeschränkt zu: Gender hat nicht mehr das Zeug zu einem neuen turn im Verdrängungswettbewerb aktueller Konzepte, gehört aber sicher in die Geschichte der kulturellen Wenden, die man ja nicht nur prophetisch in die Zukunft entwerfen muss, sondern auch historisch rekapitulieren kann. Mein Punkt ist aber ein anderer: Ob aktuell oder passé, Gender erschöpft sich keinesfalls in seiner Erscheinungsform als turn. Im Fall von Gender haben wir es nicht mit der kurzfristigen Aktualität einer konzeptuellen Wende zu tun, sondern mit einem tiefgreifenden und langfristigen kulturellen Wandel. Niemand wird in Frage stellen, das sich die westliche Welt in Bezug auf das Verhältnis der Geschlechter zwischen den 1970er Jahren und heute grundlegend verändert hat. Ähnliches könnte man auch vom ,postcolonial turn' sagen, der von Bachmann-Medick in ihre Liste aufgenommen wurde, und ebenso von der Erinnerungsforschung, dem Ecocriticism, dem animal turn, dem disability turn etc. Auf diese Entwicklung geht Bachmann-Medick am Rande ein. Sie beobachtet eine „Disziplinenaufsplitterung [...] in Forschungsfelder und Subdiziplinen wie queer studies, postcolonial studies, diability studies, whiteness studies, border studies, trauma studies usw." Darin sieht sie - und hier entspricht ihre Diagnose derjenigen Hartmut Böhmes - eine typische Erscheinung der amerikanischen Cultural Studies, die aber mit ,turns' in ihrem Sinne nichts zu tun hat (Bachmann-Medick 2014: 413). Neben den turns, die der Profilierung neuer Forschungsrichtungen dienen und den für die Disziplinen nötigen Theorie-Wandel beschleunigen, gibt es also einen gesellschaftlichen Kultur- und Bewusstseinswandel, der sowohl außerhalb der Universitäten stattfindet, als auch innerhalb, wo er sich in neugeschaffenen Subdisziplinen niederschlägt. Wie entsteht ein solcher Kulturwandel? Ich versuche eine einfache Antwort: Er entsteht, wenn etwas, das bislang implizit geblieben ist, plötzlich zum Gegenstand expliziter Diskurse und Debatten wird. Ein Kulturwandel geht mit Thematisierungsschüben einher, die eine irreversible Wende des kulturellen, historischen oder moralischen Bewusstseins einer Gesellschaft anzeigen und einleiten. Er beginnt mit einer neuen Sprache und einem neuen Denken über an sich altbekannte und allgemein relevante gesellschaftliche Phänomene. Solcher Kulturwandel entsteht durch das Zusammenwirken politischer, sozialer, medialer und epistemologischer Veränderungen, an dem auch der wissenschaftliche Diskurs einen wichtigen Anteil hat. Allgemein ins Bewusstsein tritt 
dieser Wandel nämlich erst mit einer neuen Metasprache, die inn zum Gegenstand von Debatten, Diskursen, neuen kulturellen Praktiken und kollektiven Ansprüchen macht. Meist werden dabei terminologische Differenzierungen eingeführt, die es bisher nicht gab. Erst im Lichte solcher Unterscheidungen kann man einen neuen Blick auf bekannte Phänomene werfen. So beruht der Gender-Diskurs auf der neuen Unterscheidung von sex und gender, die Erinnerungsforschung auf der neuen Unterscheidung zwischen individuellem und kollektivem Gedächtnis, sowie der Einführung des Begriffs ,Trauma', die disability studies beruhen auf Unterscheidungen wie disability, impairment und handicap, der Ecocriticism auf einer Auflösung der Opposition Natur/Kultur und die animal studies auf einer neuen Kategorisierung des Lebendigen jenseits des Anthropozentrismus.

Mit einer solchen Begriffsarbeit entsteht zugleich eine neue Perspektive und Sensibilität, die eine neue historische Stufe einleitet und einen Bewusstseinsprozess begleitet, der keineswegs von Allen vollzogen wird, aber irreversibel ist. Die Welt wird auf diese Weise komplexer, man muss immer wieder dazulernen, umdenken und vor allem auch umfühlen. Die anglo-amerikanischen Länder sind seit den 1960er und 1970er Jahren in dieser Geschichte kultureller Selbstaufklärung führend und tonangebend. Führend ist z.B. die Rolle der USA für den Gender-Diskurs oder die Rolle Großbritanniens für die postkoloniale Perspektive. Das Interessante an diesen Entwicklungen ist aber, dass sie nicht auf diese Länder beschränkt blieben, sondern globale Ausmaße erreichten und nicht zuletzt auch eine wichtige Rolle auf der Agenda der internationalen Kulturwissenschaften spielen, die diese Wirkung verstärken, indem sie dafür einen wichtigen ,Transmissionsriemen' und Resonanzboden bilden.

Hier eine erste kurze Zwischenbilanz: Im Gegensatz zu den, turns' entfalten die Themen kultureller Wenden ihre Bedeutung nicht nur innerhalb akademischer Fächer, sondern haben auch ein Leben außerhalb der Universitäten, weshalb wir ihre Dynamik schwer kontrollieren und auf ihre (Ab-)Laufzeit auch keinen direkten Einfluss nehmen können. Das macht sie für viele Theoretiker suspekt; diese Themen gelten manchen als unsachlich, politisiert oder auch trivial, weil es nicht immer einfach ist, den jeweiligen akademischen Diskurs säuberlich von der Dimen- sion kultureller Praktiken und gesellschaftlicher Debatten abzulösen.

\section{Herausforderungen, Frage- stellungen, Perspektiven, Schlüsselbegriffe}

$\mathrm{Zu}$ den Herausforderungen der Kulturwissenschaften gehört, dass sich ihre Perspektiven ständig verschieben. Ehemalige Leitbegriffe wie ,Subjekt', ,Nation', ,Geschichte', ,Natur' oder ,Tradition' erweisen sich als alles andere als stabil. Dafür tun sich permanent neue Problemfelder auf, die zur Bearbeitung anstehen und für die es noch keine etablierte Fachkompetenz und vorgängige Begriffe gibt. Durch ihre Umwandlung in Kulturwissenschaften haben die Literaturwissenschaften nicht nur eine Grenzerweiterung erfahren, sondern auch eine notorische Grenzerweichung. Das ist ein Problem, für das es keine Lösung gibt. Als Literaturwissenschaftlerinnen kümmern wir uns inzwischen intensiv um Dinge, über deren Ausschluss wir uns ehemals definiert hatten. Wir sind überall anschlussfähig geworden, aber wir haben dafür Schwierigkeiten mit der Ausschlussregel, wenn es um die Definition des Gegenstandsbereichs unserer ehemals philologischen Fächer geht. Ein Beispiel dafür ist die Arbeit am Fiktionsbegriff und seinen Gegenbegriffen wie Wahrheit, das Reale, Evidenz, oder das Historische, auf die ich hier näher eingehen möchte.

Einige Kolleginnen und Kollegen haben das Problem erkannt und arbeiten energisch an einer Grenzkontrolle zwischen Literaturwissenschaft und Kulturwissenschaft. Zu ihnen gehört Andreas Kablitz (2012), der sehr genau angeben kann, was zur Literaturwissenschaft gehört und was nicht. Ich zitiere aus seinem Buch mit dem programmatischen Titel: Kunst des Möglichen. Eine Theorie der Literatur: Gender Studies sind eine "perspektivenreiche thematische Fokussierung von Literaturwissenschaft, aber sie begründen kein eigenes Konzept von Literatur. [...] Geschlechterdifferenz konstituiert nicht Literatur" (Kablitz 2012: 263). Der Gegenstand und die Aufgabe der Literaturwissenschaft bestehen also darin, das zu untersuchen, was Literatur konstituiert. Die Cultural 
Studies machen es sich in diesem Punkt zu einfach, denn sie machen das, was Gegenstand der Untersuchung sein sollte, zu ihrer Prämisse. Diese Prämisse, so Kablitz, besteht in der "stillschweigenden Annahme [...], dass Literatur und Kultur in der Tat strukturell einander ähnlich, wo nicht identisch sind." Die Vorstellung, Literatur sei ein Paradigma von Kultur und umgekehrt hält Kablitz nicht für ein tragfähiges wissenschaftliches Fundament. Deshalb arbeitet er die spezifischen Merkmale poetischer Rede heraus, "die sie von anderen Diskurstypen unterscheidet" und definiert Literatur als "Kunst des Möglichen" (ebd.: 264). Seine Definition von Literatur kann an eine lange Genealogie der Fiktionstheorien anknüpfen. Das ist ein erfreulich klarer Standpunkt, aber wollen wir uns wirklich so rigoros einschränken lassen? Heute beschäftigt Literaturwissenschaftler vieles, was über die Kunst des Möglichen hinausgeht und sogar traditionell auf der anderen Seite der Fiktion angesiedelt war, womit es grundsätzlich jenseits des Gegenstandsbereichs der Literaturwissenschaften verblieb. Ein gutes Beispiel dafür ist das Thema des Konstanzer Graduiertenkollegs: ,Das Reale in der Kultur der Moderne' (2010ff).

Wenn wir heute über, das Reale' nachdenken, entfernen wir uns von einem mystischen Faszinations-Begriff des Realen, wie inn Hofmannsthal um 1900 in seinem Chandos-Brief verwendete, aber auch von einem katastrophischen Faszinations-Begriff des Realen, wie inn Alain Badiou (2006) ins Spiel gebracht hat, als er die Essenz des gewalttätigen 20. Jahrhunderts als, Passion für das Reale' definierte. Der neue kulturwissenschaftliche Begriff des Realen entfernt sich auch ein Stück weit von den großen philosophischen Konstruktionen, in die dieser Begriff eingespannt war, wie zum Beispiel Lacans bedeutende Triangulierung des Realen, Symbolischen und Imaginären. ${ }^{1}$ Der neue Sammelbegriff des Realen fasst all das zusammen, was traditionell der Symbolisierung entgeht und steht etwa im Konstanzer Konzept für so unterschiedliche Dinge wie Wirklichkeit, Wahrheit, Evidenz, das Neue, Anomalie, Schock und Trauma, Gewalt, Körper, Sinnlichkeit, Seinsdichte, Unmittelbarkeit, Materialität,

1 Diese Trias kehrt bei Wolfgang Iser wieder in seinem Buch: Das Fiktive und das Imaginäre: Perspektiven literarischer Anthropologie (1991).
Reinheit, Fakten, das Unsagbare, Katastrophen, Offenbarung und das Heilige. Ich habe hier die Ersatzbegriffe des Realen, wie sie im Konstanzer Antragstext vorkommen, einmal systematisch aufgelistet, um zu zeigen, dass dieser Begriff, der eigentlich eine Grenze der Literaturwissenschaft markieren sollte, selbst notorisch schwer in Grenzen zu halten ist. In diesen verschiedenen Erscheinungsformen wird das Reale, das sich einer Symbolisierung versperrt, als eine relevante Bezugsgröße und auch Komponente von literarischen Texten wiederentdeckt. Faktische Elemente sind längst in die Struktur literarischer Texte eingedrungen: als Spur des Historischen in der Literatur, als Anteil des Dokumentarischen in fiktionalen Formaten oder als die Wiederkehr der Begriffe ,Erinnerung' und ,Erfahrung'. Vor allem aber wird mit dem Begriff des Realen die Frage der Referenz in die Literaturwissenschaft zurückgeholt, die bislang axiomatisch ausgeschlossen war. Literatur war ja definiert worden als das, was seine Geltung jenseits der Wirklichkeit hatte. Die kulturwissenschaftliche Öffnung der Literaturwissenschaft zeigt sich in der Veränderung ihres Vokabulars. Diese Öffnung kommentiert auch Bachmann-Medick mit einem antizipierenden Blick auf die Zukunft neuer Wenden: "Entscheidend wird aber sein, dieses Vokabular ausdrücklich rückzukoppeln an die Reflexion von Inhalten, historischen Bezügen und an einen neu zu bestimmenden Anspruch auf Referentialität." (Bachmann-Medick 20014: 414) Diese "Wiedergewinnung von Referentialität" ist verbunden mit einer "(veränderten) Rückkehr des poststrukturalistisch verabschiedeten Subjekts, des ,Handelnden', oder des ,Autors', aber auch" mit der "gegenwärtigen Aufwertung von ,Materialität" und der "Nähe zu den Phänomenen", ja zur "Evidenz" (ebd.).

Eine weitere Grenzüberschreitung, die die Literaturwissenschaft mit ihrer Verwandlung in Kulturwissenschaft in den letzten Jahrzehnten erfahren hat, ist der Schritt von der abstrakten Kategorie ,Text' zur Kategorie ,Buch'. Vom Text kam man zu den Texten, und von dort aus trat man ein ins Labyrinth der Intertextualität. Heute kommt man vom Text zum Objekt Buch, zur Frage nach der materiellen Existenzform von Texten, zur Produktion von Wissen und zu den Ordnungen des Wissens, zu kulturellen Praktiken des Übersetzens und Sammelns, der Katalogisie- 
rung und der Kanonisierung. Die Wahrnehmung der Literatur hat eine Außenseite bekommen. Sie zeigt sich eingebettet in veränderte mediale Kontexte und kulturelle Praktiken und damit in eine übergreifende Geschichte der Wissensproduktion. Das ist nur ein weiterer Beleg für die notorische Entgrenzung unseres einst so klar definierten Gegenstands.

\section{Zwei Beispiele kulturwissen- schaftlicher Entgrenzung}

Ich möchte hier noch zwei Beispiele kulturwissenschaftlicher Forschung anführen, die programmatisch Grenzen überschreiten und außer Kraft setzen. Das eine Beispiel kommt aus der Literaturwissenschaft, das andere aus der Geschichtswissenschaft. Beide Beispiele können auch als Ergänzung jener Felder verstanden werden, die Hartmut Böhme in seinem Beitrag exemplarisch vorschlägt, wobei es mir vornehmlich darum gehen wird, unterschiedliche Zugangsweisen innerhalb der Kulturwissenschaften kenntlich zu machen. Albrecht Koschorke ist ein Germanist, der seine Form der Kulturwissenschaft als, Grundlagenforschung' versteht. Wie in den Naturwissenschaften, die in Angewandte Forschung und Grundlagenforschung aufgeteilt sind, fordert er eine ähnliche Einteilung für die Kulturwissenschaften. Während die angewandte Forschung sich mit Textinterpretationen und Case Studies beschäftigt, untersuchen andere die Grundlagen dessen, was menschliches Zusammenleben in der Kultur ausmacht und zusammenhält. ${ }^{2}$ In diesem Sinne hat er sich einer exemplarischen Domäne kulturwissenschaftlicher Grundlagenforschung, nämlich des Erzählens, angenommen und es zum Gegenstand seiner Forschung gemacht. In seiner gewichtigen Studie Wahrheit und Erfindung. Grundzüge einer Allgemeinen Erzähltheorie beginnt er deshalb auch mit der Universalität des Phänomens des Erzählens, einer Anthropologie des ,homo narrans' (Koschorke 2012: 9).

2 Die Arbeitsform der Text-Interpretation hält Koschorke für überholt, wie er in einer Stellungnahme zur aktuellen Situation seines Fachs in der letzten Ausgabe der Deutsche Vierteljahrsschrift für Literaturwissenschaft und Geistesgeschichte 89 (2015) betont.
Erzählen hat ganz unterschiedliche Funktionen, es erscheint auf dieser allgemeinen Ebene als spielerischer Zeitvertreib, als Angstbewältigung oder Sinnkonstruktion. Koschorke ist aufgefallen, dass das Erzählen, das seinen privilegierten Ort in der Literatur hatte, aus diesem Reservat längst ausgebrochen ist, was mit einer Aufhebung "der gesellschaftlichen Arbeitsteilung und der damit einhergehenden Aufgliederung in unterschiedliche Rationalitätsbereiche" zusammenhängt. (Koschorke 2012: 19) Da das Erzählen alle Grenzen gesprengt hat, kann Koschorke es über diese Grenzen hinweg nachverfolgen, und schließlich auch noch über die einer, transgenerischen Narratologie' (Ansgar Nünning). Der Begriff Erzählung, so stellt er fest, ist „zu einer der transdisziplinär erfolgreichsten und expansionsfreudigsten literaturwissenschaftlichen Kategorien geworden". (ebd.) In unterschiedlichen Disziplinen ist auch von einem ,narrative turn' die Rede, doch Koschorke schreibt sich diesen turn nicht auf seine Fahnen. Er ist nicht forschungsstrategisch an einem neuen Paradigma interessiert, das er für alle verbindlich institutionalisieren will, sondern vielmehr an der Ubiquität des Phänomens Erzählen selbst, das er von der Alltagskommunikation, der Literatur und der Unterhaltung in den Massenmedien bis in die Rechtsfiktionen, "Gründungsmythen und Lebenslügen ganzer Gesellschaften" verfolgt (ebd., 19f.).

Im Grunde geht es Koschorke dabei aber gar nicht um das Erzählen. Man hat inm zum Vorwurf gemacht, dass er als Germanist in seinem dicken Buch über das Erzählen auf keinen einzigen literarischen Text, auf keinen Film, auf keine konkrete Geschichte eingegangen ist (Groebner 2013). Sein Thema ist nämlich gar nicht das Erzählen, sondern das Narrativ. Beide haben nicht viel miteinander gemein. Das Erzählen erfolgt von Mensch zu Mensch und ist ein in Raum und Zeit eingebetteter anschaulicher, einfallsreicher und kommunikativer Sprechakt, der aber auch in vielen Medien spannungsvoll und anziehend präsentiert wird. Das Narrativ verhält sich zum Erzählen wie die Blaupause auf dem Reißbrett des Architekten zum wirklichen Gebäude. Das Narrativ ist eine abstrakte Struktur, die zugleich eine Form der Sinngebung ist, in die Handlungen, politische Ziele, Geschichtsbilder und menschliche Erfahrungen gepresst werden. Es ist der rote Faden, der eine klar definierte Auswahl heterogener Ereig- 
nisse zusammenhält und ihnen mit Anfang, Mitte und Ende zugleich Struktur, Sinn und Ziel verleiht. Aristoteles' Wort für eine solche Geschichte war ,Mythos'. Koschorke geht es um diese magische Wirkung des Narrativs bzw. Mythos, der nicht nur Spannungsaufbau produziert und Entlastung von Spannungen ermöglicht, sondern Menschen auch verbindlich zusammenbinden und Institutionen legitimieren kann. Narrative bzw. Mythen sind in vielen Lebensbereichen wirksam und steuern individuelles und kollektives menschliches Handeln. Es geht bei Koschorke also gerade nicht um die poetische Textur des Erzählens, die dem Literarischen als ein Alleinstellungsmerkmal anhaftet und poetische Rede konstituiert, sondern um einen elementaren Modus der Sinngebung, der wie er so schön sagt - "der Literatur entlaufen ist" und nun in unterschiedlichen Bereichen der Kultur identifiziert und analysiert werden kann. In der allgemeinsten Definition von Koschorke (2012: 25) wird das Erzählen gar „zum Organon einer unablässigen kulturellen Selbsttransformation". Es ist das zentrale Instrument des, worldmaking', das im doppelten Sinne entgrenzt ist, zum einen, weil es alle möglichen kulturellen Felder durchzieht und zum anderen, weil es als eine Energie aufgefasst wird, die beständige Veränderungen auslöst.

Als zweites Beispiel einer kulturwissenschaftlichen Untersuchung, die bewusst auf Grenzüberschreitung angelegt ist, möchte ich hier die Arbeiten des Kulturhistorikers Karl Schlögel anführen. Ich beziehe mich dabei konkret auf die kleine Studie Archäologie des Kommunismus, in der er sein Forschungsprogramm der letzten Jahre zusammengefasst und pointiert hat. Im Zentrum stehen dabei die Jahre des Terrors unter Stalin im Russland der 1930er Jahre, denen er sich in immer neuen Anläufen anzunähern versucht. Er entwickelt dafür eine Archäologie nicht nur der Denkformen, sondern auch der mikrohistorischen Strukturen, um diese Epoche willkürlicher und entfesselter Gewalt so genau und umfassend wie nur irgend möglich zu beschreiben. Während die Geschichtsschreibung bzw. Geschichtserzählung notwendig einer linearen Abfolge verpflichtet ist, verschreibt sich Schlögels Darstellung dem Ideal einer umfassenden Gleichzeitigkeit. Seine Form der Geschichtsschreibung setzt sich zum Ziel, die geschichtliche Totalität in der beschreibenden Rekonstruktion zu vergegenwärtigen. Diese
Idee seiner ,histoire totale' setzt er u.a. in der Manier eines, material turn' um. Die materiellen Hinterlassenschaften der Geschichte, die "von der Brandung des Lebens ans Ufer geworfen" wurden, werden von ihm minutiös registriert und aufgesammelt. Er gibt sich allerdings mit dem Einsammeln des Schutts der Geschichte nicht zufrieden. "Wie müssten die Sonden angesetzt werden?" fragt er. "Wie navigiert man durch die Lebenswelt von Generationen von Sowjetmenschen, ohne dem Zufall, der Willkür, einer subjektiven Obsession oder Idiosynkrasie aufzusitzen?" (Schlögel 2014: 55) Schlögel hat den Ehrgeiz, sich frei zu machen von den Irrwegen der Historiker: dem Weg des Exemplarischen, dem Weg der Hierarchisierung von Bedeutsamkeit oder der Reduktion von Komplexität durch ein Narrativ. Sein Vorbild ist der Archäologe, der gräbt, ohne schon im Voraus zu wissen, wo er fündig wird und wohin die Entdeckungsreise geht. „Dabei ist es fast beliebig, womit wir beginnen, da jetzt schon klar ist, dass an jeder dieser Stellen etwas von Bedeutung ausgemacht werden kann." (ebd., 57) Dieser Historiker erzählt nicht, sondern zählt auf. Seine Untersuchungs-Einheiten nennt er ,Topoi', die nicht in eine Hierarchie oder Linie zu bringen sind, z.B. der Grenzübertritt, der Schwarzmarkt als Pendant zur Planwirtschaft, Packpapier und Müll, Museum und Diorama, die Nippesfiguren, "die Interieurs als wahre Kampfzonen der großen geschichtlichen Auseinandersetzungen" (ebd., 63), der Inhalt von Kommoden-Schubladen, das Klavier, das Krematorium, das Sanatorium, die Eisenbahn, das Vorzimmer mit seiner wattierten Tür, die öffentlichen Toiletten, die Datscha, die Palme usw. usw. Hinter diese Aufzählung kann man keinen Punkt setzen, denn die Liste kann niemals vollständig sein.

Die Aufzählung der Topoi oder Gemeinplätze der sowjetischen Zivilisation ist für Schlögel die einzig angemessene Form der Annäherung an die historische Wirklichkeit. Sein Credo dieser grenzenlosen Form der Kulturwissenschaft lautet: ,Es gibt keinen Gegenstand, an dem sich nicht - wie in einem Prisma - die ganze Geschichte erzählen ließe.' Auch er verzichtet also nicht auf das Erzählen, aber es fügt sich bei ihm mit Sicherheit nicht zu einem Narrativ der Epoche, die er untersucht. Es gibt deshalb auch nicht eine Erzählung, sondern eine unabschließbare Vielfalt von Erzählungen, deren Zahl gegen unendlich geht. Jede 
davon rekonstruiert verflochtene Mikrowelten. Sie markiert einzelne Fäden eines Gewebes, die man ein Stück weit verfolgen kann, ohne dass man aber das Gesamtgewebe von irgend einem Punkt aus in seinem Zusammenhang und seiner Gänze übersehen könnte. Die überwältigende Anhäufung von Material und die damit einhergehende Überblicklosigkeit ist hier Prinzip, die Annäherung an das Chaos der vergangenen Wirklichkeit ist die Methode dieser grenzenlosen Kulturwissenschaft, die in der sinnlichen Annäherung mithilfe der Topoi zugleich alle abstrakten Synthesen dekonstruiert.

Die Erzähltheorie von Albrecht Koschorke und die histoire totale von Karl Schlögel sind zwei Beispiele einer programmatisch grenzüberschreitenden Kulturwissenschaft, die dabei allerdings gegensätzliche Wege gehen. Koschorke geht es um radikale Reduktion und höchste Generalisierung; er sieht von allen Einzelheiten ab, um die allgemeine Funktion von Narrativen als Landkarten, Raster, Orientierungsprogramme, kurz: als Mittel der Erzeugung von Bedeutung, Relevanz und damit auch von Realität herauszuarbeiten. Schlögel vermeidet umgekehrt jegliche Reduktion und Selektion; er geht von der unendlichen Vielzahl konkreter Details aus und strebt eine asymptotische Annäherung an die Realität an: ein ebenso faszinierendes wie fantasmatisches Projekt.

\section{Quo vaditis, Kulturwissenschaften?}

Inzwischen befinden sich die Kulturwissenschaften, wie uns Doris Bachmann-Medick mitteilt, "nicht mehr in der Hochphase eines Wende-Enthusiasmus", der durch permanente Neuorientierung zur Desorientierung führt. Jeder turn inszeniert und inauguriert ein neues Konzept mit einer klaren Ausschlusslogik nach dem Motto: „Ein Kapitel ist geschlossen, ein anderes geöffnet." Warum eigentlich? Eine Korrektur dieses Problems könnte darin bestehen, die neuen Impulse nicht in der Rhetorik einer fortgesetzten Ersetzungslogik zu sehen, sondern als willkommene Umperspektivierungen im Sinne einer allgemeinen Erweiterungslogik des Wissens und Forschens. Es gibt nämlich keinen ersichtlichen Grund, warum sie überhaupt als Folge verzeitlicht werden müssen und nicht nebeneinander stehen können als produktive Konzepte und einander ergänzende Forschungsperspektiven. Paradigmenwechsel folgen dem Druck der Selbstanpreisung eines Wissenschaftsmanagements, das stets Neues verlangt und dafür auch bereit ist, das Bestehende ad acta zu legen. Neue Perspektiven dagegen haben keinen solch ausschließenden Charakter und lassen sich nach Belieben miteinander verbinden. In den Kulturwissenschaften werden aber - wie wir gesehen haben - nicht nur neue Impulse der Theorie aufgenommen, sie sind auch der Lebenswelt gegenüber enorm porös und hellhörig für gesellschaftliche Veränderung. Für alle wissenschaftlichen Disziplinen gilt, dass sie nicht nur in ihre autonomen Fachkulturen, sondern auch in die Gesellschaft eingebettet sind. Die Kultur ist das übergreifende und umfassende Erzeugungssystem, innerhalb dessen auch alle Wissenschaften ihren Platz haben. ${ }^{3}$ Aber anders als in den Naturwissenschaften spricht man in den Kulturwissenschaften nicht von außen, sondern immer schon von innen. Hier ist die Betrachterin, ob sie sich das klar macht oder nicht, immer schon mit im Bild. Die Kulturwissenschaften unterscheiden sich deshalb von anderen Fächern dadurch, dass die Grenze zu ihrem Gegenstand besonders durchlässig ist. Sie beobachten genau und reagieren dabei auf die sie umgebende Welt, die zugleich ihr Gegenstandsbereich ist. Ihr Auftrag ist, verallgemeinernd gesagt, die Selbstbeobachtung und Selbstthematisierung der Kultur, deshalb sind die Kulturwissenschaften anders als andere Disziplinen von vorn herein auf Grenzerweichung, Grenzerweiterung und Grenzüberschreitung angelegt. Sie lassen sich ebenso wenig feststellen und eingrenzen wie die Kultur selbst, denn genau wie Koschorkes Konzept des Erzählens sind sie ,das Organon einer unablässigen kulturellen Selbsttransformation'.

3 ,Science in Culture' heißt die Formel für diese Einsicht und ist zugleich der Name einer neuen Metadisziplin für die Naturwissenschaften. 


\section{Literaturverzeichnis}

Bachmann-Medick, Doris (2014): Cultural Turns. Neuorientierung in den Kulturwissenschaften. Reinbek bei Hamburg: Rowohlt, 5. Aufl.

Badiou, Alain (2006): Das Jahrhundert. Zürich/Berlin: Diaphanes.

Groebner, Valentin (2013): „Überschwängliche Ausnüchterung. Albrecht Koschorke erzählt von sich". In: Merkur. Zeitschrift für europäisches Denken 768 (Mai), S. 451-456.

Iser, Wolfgang (1991): Das Fiktive und das Imaginäre: Perspektiven literarischer Anthropologie. Frankfurt a.M.: Suhrkamp.
Kablitz, Andreas (2012): Kunst des Möglichen. Eine Theorie der Literatur. Freiburg: Rombach Verlag.

Koschorke, Albrecht (2012): Wahrheit und Erfindung. Grundzüge einer Allgemeinen Erzähltheorie. 2. Aufl. Frankfurt a.M.: S. Fischer.

Koschorke, Albrecht (2015): Die Germanistik auf dem Weg zu einem kleinen Fach. In: Deutsche Vierteljahrsschrift 89/4, S. 587-594.

Schlögel, Karl (2003): Im Raume lesen wir die Zeit. Über Zivilisationsgeschichte und Geopolitik. München: Hanser.

Schlögel, Karl (2014): Archäologie des Kommunismus oder Russland im 20. Jahrhundert. Ein Bild neu zusammensetzen. München: Carl Friedrich von Siemens Stiftung. 